\title{
DYNAMIC FEEDBACK EQUIVALENCE OF NONLINEAR SYSTEMS ON TIME SCALES
}

\author{
Zbigniew Bartosiewicz ${ }^{*, 1}$ Ewa Pawłuszewicz ${ }^{*, 1}$
}

\author{
* Institute of Mathematics and Physics \\ Biatystok Technical University \\ Wiejska 45A, 15-351 Biatystok, Poland
}

\begin{abstract}
The problem of dynamic feedback equivalence of nonlinear control systems on time scales is studied. Time scale is a model of time. Two most important cases are the real line (continuous time) and the set of integers (discrete time). Control systems on time scales include continuous-time and discrete-time systems. The delta algebra of a nonlinear control system is introduced. The main result says that two systems defined on a time scale are dynamically feedback equivalent if and only if their delta algebras are isomorphic. This is an extension of continuous-time and discrete-time versions. Copyright (c)2005 IFAC
\end{abstract}

Keywords: Behaviour; control system; differential equations; continuous-time systems; discrete-time systems; dynamic feedback; feedback linearization; nonlinear control systems.

\section{INTRODUCTION}

The concept of dynamic feedback equivalence was first introduced and investigated by Jakubczyk (1992 and 1993) for continuous-time systems. This concept allowed for a simple and elegant definition of dynamic feedback linearization, which was earlier studied by many authors, as for example Marino (1992) and Fliess et al. (1992 and 1993) See also Pomet (1995) for more references on dynamic linearization. Jakubczyk has shown that two nonlinear continuous-time control systems are dynamically feedback equivalent if and only if their differential algebras are isomorphic. The differential algebra of a continuous-time system is a certain algebra of real functions together with a differential operator associated with the dynamics of the system.

\footnotetext{
1 This work has been supported by KBN under Bialystok Technical University grant No W/IMF/1/04
}

Jakubczyk's result was transferred to the discretetime case by Bartosiewicz et al. (1994). In that new setting dynamic transformations depended on future states and controls and the difference operator associated with the discrete-time system appeared instead of the differential operator. The corresponding theorem stated that two discretetime systems are dynamically feedback equivalent if and only if their difference algebras are isomorphic.

Calculus on time scales, developed by Hilger (1988), unifies differential calculus on the real line and calculus of finite differences. There is, in fact, whole spectrum of different time scales which serve as models of time; continuous time and discrete time are just two most important cases. Dynamical systems on time scales were studied by Bohner and Peterson (2001). Basics of theory of linear control systems were developed by Bartosiewicz and Pawłuszewicz (2004a and 2004b). There were other attempts to unify discrete and continuous in control theory. Let us 
mention Monaco and Normand-Cyro (1995) and Goodwin et al. (2001). Time scales, however, besides unification allow to consider time that is partly continuous and partly discrete. This could be used to model certain phenomena in biology, where one can observe different stages of life of some species.

In this paper we study dynamic feedback equivalence of nonlinear control systems on time scales. We give a characterization of this property which unifies continuous-time and discrete-time cases. We introduce the delta operator associated with a system on time scale. In general, it is neither differential nor difference operator. It is shown that two systems on time scales are dynamically feedback equivalent if and only if their delta algebras are isomorphic. We consider only homogeneous time scales for which time-invariant systems may be defined. Nonhomogeneous case will require some modifications. This will be studied in future papers.

Dynamic output equivalence for nonlinear discretetime systems was studied by Bartosiewicz and Pawłuszewicz (1998) and Pawłuszewicz and Bartosiewicz (1999). The difference universe of a nonlinear system with output was used instead of the difference algebra to characterize this property.

\section{CALCULUS ON TIME SCALES}

A time scale $\mathbb{T}$ is an arbitrary nonempty closed subset of the set $\mathbb{R}$ of real numbers. It serves as a model of time. The standard cases comprise $\mathbb{T}=\mathbb{R}, \mathbb{T}=\mathbb{Z}$ and $\mathbb{T}=h \mathbb{Z}$ for $h>0$. Another interesting time scale is $P_{a, b}=\bigcup_{k=0}^{\infty}[k(a+$ $b), k(a+b)+a]$. Here time is partly continuous and partly discrete. Bohner and Peterson (2001) use this time scale to describe evolution of certain physical and biological systems (e.g. RLC circuit and population of cicada). To see more possibilities, imagine water dripping from a faucet. This discrete-time phenomenon often changes its nature to continuous-time as water starts to flow continuously at some moment. The time scale here would be a sequence of points (finite or infinite?) followed by a half-line.

We assume that $\mathbb{T}$ is a topological space with the relative topology induced from $\mathbb{R}$. For $t \in \mathbb{T}$ we define

- the forward jump operator $\sigma: \mathbb{T} \rightarrow \mathbb{T}$ by $\sigma(t):=\inf \{s \in \mathbb{T}: s>t\}$

- the backward jump operator $\rho: \mathbb{T} \rightarrow \mathbb{T}$ by $\rho(t):=\sup \{s \in \mathbb{T}: s<t\}$

- the graininess function $\mu: \mathbb{T} \rightarrow[0, \infty)$ by $\mu(t):=\sigma(t)-t$.

If $\sigma(t)>t$, we say that $t$ is right-scattered, while if $\rho(t)<t$ we say that $t$ is left-scattered. Points that are right- and left-scattered at the same time are called isolated. If $t<\sup \mathbb{T}$ and $\sigma(t)=t$, then $t$ is called right-dense; if $t>\inf \mathbb{T}$ and $\rho(t)=t$ then $t$ is called left-dense. Points that are rightand left-dense at the same time are called dense.

Finally we define the set

$$
\mathbb{T}^{k}:= \begin{cases}\mathbb{T} \backslash(\rho(\sup \mathbb{T}), \sup \mathbb{T}] & \text { if } \sup \mathbb{T}<\infty \\ \mathbb{T} & \text { if } \sup \mathbb{T}=\infty\end{cases}
$$

Example 2.1.

- If $\mathbb{T}=\mathbb{R}$ then for any $t \in \mathbb{R}, \sigma(t)=t=\rho(t)$; the graininess function $\mu(t) \equiv 0$.

- If $\mathbb{T}=\mathbb{Z}$ then for every $t \in \mathbb{Z}, \sigma(t)=t+1$, $\rho(t)=t-1$; the graininess function $\mu(t) \equiv 1$.

Definition 2.2. Let $f: \mathbb{T} \rightarrow \mathbb{R}$ and $t \in \mathbb{T}^{k}$. Delta derivative of $f$ at $t$, denoted by $f^{\triangle}(t)$, is the real number (provided it exists) with the property that given any $\varepsilon$ there is a neighborhood $U=(t-\delta, t+$ $\delta) \cap \mathbb{T}$ (for some $\delta>0$ ) such that

$\left|(f(\sigma(t))-f(s))-f^{\triangle}(t)(\sigma(t)-s)\right| \leq \varepsilon|\sigma(t)-s|$

for all $s \in U$. Moreover, we say that $f$ is delta differentiable on $\mathbb{T}^{k}$ provided $f^{\triangle}(t)$ exists for all $t \in \mathbb{T}^{k}$

We shall often drop the word "delta" and talk just only about derivatives and differentiability, if this does not lead to confusion.

In general case forward jump operator $\sigma$ is not delta differentiable.

Assume that $f, g: \mathbb{T} \rightarrow \mathbb{R}$ are delta-differentiable at $t \in \mathbb{T}^{k}$. Then:

(1) $(f+g)^{\triangle}(t)=f^{\triangle}(t)+g^{\triangle}(t)$;

(2) $(a f)^{\triangle}(t)=a f^{\triangle}(t)$ for any constant $a$;

(3) $(f g)^{\triangle}(t)=f^{\triangle}(t) g(t)+f(\sigma(t)) g^{\triangle}(t)=$ $f(t) g^{\triangle}(t)+f^{\triangle}(t) g(\sigma(t))$

(4) if $g(t) g(\sigma(t)) \neq 0$ then $\left(\frac{f}{g}\right)^{\triangle}(t)=\frac{f^{\triangle}(t) g(t)-f(t) g^{\triangle}(t)}{g(t) g(\sigma(t))} ;$

(5) in general $(f \circ g)^{\triangle} \neq\left(f^{\triangle} \circ g\right) g^{\triangle}$.

For proofs of these and other facts presented in this section see e.g. (Bohner and Peterson, 2001).

\section{Remark 1.}

- If $\mathbb{T}=\mathbb{R}$, then $f: \mathbb{R} \rightarrow \mathbb{R}$ is delta differentiable at $t \in \mathbb{R}$ iff

$$
f^{\triangle}(t)=\lim _{s \longrightarrow t} \frac{f(t)-f(s)}{t-s}=f^{\prime}(t) .
$$

i.e. iff $f$ is differentiable in the ordinary sense at $t$.

- If $\mathbb{T}=\mathbb{Z}$, then $f: \mathbb{Z} \rightarrow \mathbb{R}$ is always delta differentiable at every $t \in \mathbb{Z}$ with

$$
f^{\triangle}(t)=f(t+1)-f(t) .
$$


Thus delta derivative is then the usual forward difference operator.

Example 2.3. The delta derivative of $t^{2}$ is $t+\sigma(t)$. This means that the second delta derivative of $t^{2}$ may not exists.

The delta-derivative of $\frac{1}{t}$ is $\frac{-1}{t \sigma(t)}$.

A function $f: \mathbb{T} \rightarrow \mathbb{R}$ is called regulated provided its right-sided limits exist (finite) at all right-dense points at $\mathbb{T}$ and its left-sided limits exist (finite) at all left-dense points in $\mathbb{T}$. A function $f: \mathbb{T} \rightarrow \mathbb{R}$ is called $r d$-continuous provided it is continuous at right-dense points in $\mathbb{T}$ and its left-sided limits exist (finite) at left-dense points in $\mathbb{T}$. It can be shown that

$f$ is continuous $\Rightarrow f$ is rd-continuous $\Rightarrow f$ is regulated

and that $\sigma$ is rd-continuous.

The set of functions $f: \mathbb{T} \rightarrow \mathbb{R}$ that are $n$ times delta differentiable and whose all derivatives are rd-continuous will be called of the $C^{n} r d$-class. Similarly $C^{\infty}$ will denote the class of all functions having derivatives of arbitrary order.

A continuous function $f: \mathbb{T} \rightarrow \mathbb{R}$ is called predifferentiable with (the region of differentiation) $D$, provided $D \subset \mathbb{T}^{k}, \mathbb{T}^{k} \backslash D$ is countable and contains no right-scattered elements of $\mathbb{T}$, and $f$ is differentiable at each $t \in D$. It can be proved that if $f$ is regulated then there exists a function $F$ that is pre-differentiable with region of differentiation $D$ such that

$$
F^{\triangle}(t)=f(t)
$$

for all $t \in D$. Any such function is called preantiderivative of $f$. Then indefinite integral of $f$ is defined by

$$
\int f(t) \triangle t=F(t)+C
$$

where $C$ is an arbitrary constant. Cauchy integral is defined by

$$
\int_{r}^{s} f(t) \triangle t=F(s)-F(r) \text { for all } r, s \in \mathbb{T}^{k}
$$

A function $F: \mathbb{T} \rightarrow \mathbb{R}$ is called an antiderivative of $f: \mathbb{T} \rightarrow \mathbb{R}$ provided $F^{\triangle}(t)=f(t)$ holds for all $t \in \mathbb{T}^{k}$.

It can be shown that:

$$
\begin{aligned}
& \text { - } \int_{a}^{b} f(t) g^{\triangle}(t) \triangle t= \\
& (f g)(b)-(f g)(a)-\int_{a}^{b} f^{\triangle}(t) g(\sigma(t)) \triangle t ;
\end{aligned}
$$

- if $f$ is rd-continuous and $t \in \mathbb{T}^{k}$, then $\sigma(t)$ $\int_{t}^{\sigma(t)} f(\tau) \triangle(\tau)=\mu(t) f(t)$
- if $f(t) \geq 0$ for all $a \leq t<b$, then $\int_{a}^{b} f(\tau) \triangle(\tau) \geq 0$

Remark 2. It can be shown that every rd-continuous function has an antiderivative. Moreover, if $f(t) \geq$ 0 for all $a \leq t<b$ and $\int_{a}^{b} f(\tau) \triangle \tau=0$ then $f \equiv 0$.

Example 2.4.

- If $\mathbb{T}=\mathbb{R}$, then $\int_{a}^{b} f(\tau) \Delta \tau=\int_{a}^{b} f(\tau) d \tau$, where the integral on the right is the usual Riemann integral.

- If $\mathbb{T}=\mathbb{Z}$, then $\int_{a}^{b} f(\tau) \triangle \tau=\sum_{t=a}^{b-1} f(t)$ for $a<b$.

- If $\mathbb{T}=h \mathbb{Z}, h>0$, then $\int_{a}^{b} f(\tau) \triangle \tau=$ $\sum_{t=\frac{a}{h}}^{\frac{b}{h}-1} f(t h) h$ for $a<b$.

Remark 3. An antiderivative of 0 is 1 , an antiderivative of 1 is $t$, but it is not possible to find a closed formula of an antiderivative of $t$ : antiderivative of $\frac{t^{2}}{2}$ is $\frac{t+\sigma(t)}{2}=t+\frac{\mu(t)}{2}$.

Example 2.5. If $\mathbb{T}=\mathbb{Z}$ and $a \neq 1$, then $\int a^{t} \triangle t=$ $\frac{a^{t}}{a-1}+C$, since $\left(\frac{a^{t}}{a-1}\right)^{\triangle}=\frac{a^{t+1}-a^{t}}{a-1}=a^{t}$.

\section{SYSTEMS AND ALGEBRAS}

From now on we shall assume that the time scale $\mathbb{T}$ is homogeneous, i.e. $t+\mathbb{T}:=\{t+s, s \in \mathbb{T}\}=\mathbb{T}$ for every $t \in \mathbb{T}$, and that $0 \in \mathbb{T}$. Although this assumption is not necessary, it will allow us for studying time-invariant systems.

Let us consider the following control system $\Sigma$ defined on a (homogeneous) time scale $\mathbb{T}$

$$
x^{\triangle}(t)=f(x(t), u(t)),
$$

where $t \in \mathbb{T}, x(t) \in \mathbb{R}^{n}, u(t) \in \mathbb{R}^{m}$ and $f$ is a map from $\mathbb{R}^{n} \times \mathbb{R}^{m}$ into $\mathbb{R}^{n}$. We shall assume that $f$ is of class $C^{\infty}$ and that $u$ may be delta-differentiated arbitrarily many times at every $t$. This guarantees existence and uniqueness of local solutions when we specify control $u$ and an initial condition for $x$ (see Bohner and Peterson, 2001).

By a trajectory of $\Sigma$ we mean any pair $(x, u)$ of functions defined on a subset of $\mathbb{T}$ that satisfy (1). The behavior of $\Sigma$, denoted by $\mathcal{B}(\Sigma)$, is the set of all its trajectories.

Observe that if the pair $(x, u)$ is a trajectory of $\Sigma$ and $s \in \mathbb{T}$ then the shifted pair $(\bar{x}, \bar{u})$, where $\bar{x}(t)=x(t-s)$ and $\bar{u}(t)=u(t-s)$, is also a trajectory of $\Sigma$. This means that our system is 
time-invariant and we may study only trajectories starting at time equal 0 . Another possibility would be to give up homegeneity of the time scale and to consider only local version with trajectories starting always at time equal 0 .

Let $J(m)$ denote the space of all infinite sequences $U=\left(u^{(0)}, u^{(1)}, \ldots\right)$, where $u^{(k)} \in \mathbb{R}^{m}$. We think of components of $U$ as independent variables, but if some control $u$ is chosen, the sequence $U$ will often be interpreted as the infinite jet of $u$ at time 0 .

Let $T$ be a map $\mathbb{R}^{n} \times J(m) \rightarrow J(n)$ defined by

$$
T\left(x_{0}, U\right):=X=\left(x^{(0)}, x^{(1)}, \ldots\right),
$$

where $(X, U)$ is the infinite jet at $t=0$ of a trajectory $(x, u)$ of $\Sigma$ that satisfies the initial condition $x(0)=x_{0}$. One can show that such $X$ is unique which means that $T$ is well defined. We shall also need the projection map

$P_{J(m)}: \mathbb{R}^{n} \times J(m) \rightarrow J(m), P_{J(m)}(x, U)=U$.

Following Jakubczyk (1992) and Bartosiewicz et al. (1994) we shall assume the following conditions about the system:

Condition A. For every $x, y \in \mathbb{R}^{n}$ there is at most one $u$ that satisfies the equation

$$
y=f(x, u) .
$$

Condition B. For any $x$ and $u$ the rank of the matrix

$$
\frac{\partial f}{\partial u}(x, u)
$$

is full (i.e. equal $m$ ).

Condition C. The map $\mathbb{R}^{n} \times \mathbb{R}^{m} \rightarrow \mathbb{R}^{n} \times \mathbb{R}^{n}$ : $(x, u) \mapsto(x, f(x, u))$ is proper, i.e. the inverse image of a compact set in $\mathbb{R}^{n} \times \mathbb{R}^{n}$ is a compact set in $\mathbb{R}^{n} \times \mathbb{R}^{m}$.

Let us consider two systems

$$
\Sigma: x^{\triangle}(t)=f(x(t), u(t))
$$

and

$$
\tilde{\Sigma}: \tilde{x}^{\triangle}(t)=\tilde{f}(\tilde{x}(t), \tilde{u}(t))
$$

with $x(t) \in \mathbb{R}^{n}, \tilde{x}(t) \in \mathbb{R}^{\tilde{n}}, u(t), \tilde{u}(t) \in \mathbb{R}^{m}$, defined on the same time scale $\mathbb{T}$.

Consider dynamic feedback transformations of one system into the other and vice versa

$$
\begin{aligned}
x(t) & =\phi\left(\tilde{x}(t), \ldots, \tilde{x}^{(r)}(t),\right. \\
u(t) & =\psi\left(\tilde{x}(t), \ldots, \tilde{x}^{(r)}(t), \tilde{u}(t), \ldots, \tilde{u}^{(r)}(t)\right) \\
\tilde{x}(t) & =\tilde{\phi}\left(x(t), \ldots, x^{(r)}(t),\right. \\
\tilde{u}(t) & =\tilde{\psi}\left(x(t), \ldots, x^{(r)}(t), u(t), \ldots, u^{(r)}(t)\right)
\end{aligned}
$$

These transformations depend on states, controls and their delta derivatives up to some finite order $r$. We say that two systems $\Sigma$ and $\tilde{\Sigma}$ are dynamically feedback equivalent if there are dynamic feedback transformations that transform the behavior of one system onto the behavior of the second system and vice versa, and these transformations are mutually inverse on the behaviors.

Let $A(n, m)$ denote the algebra of all $C^{\infty}$ functions

$$
\varphi: \mathbb{R}^{n} \times J(m) \rightarrow \mathbb{R}
$$

depending only on a finite number of elements in $U \in J(m)$. Let us now consider a system $\Sigma$, described by (1). Define the operator $\delta_{\Sigma}$ : $A(n, m) \rightarrow A(n, m)$ associated with $\Sigma$ by

$$
\begin{aligned}
& \left(\delta_{\Sigma} \varphi\right)(x, U):= \\
& \int_{0}^{1} \frac{\partial \varphi}{\partial x}\left(x+h \mu(0) f\left(x, u_{0}\right), U\right) d h \cdot f\left(x, u_{0}\right)+ \\
& \left.\sum_{i=0}^{\infty} \int_{0}^{1} \frac{\partial \varphi}{\partial u_{i}}\left(x, U+h \mu(0) U_{1}\right)\right) d h \cdot u_{i+1} .
\end{aligned}
$$

Remark 3.1. The delta operator has the following interpretation. Let $U(\cdot)$ be the infinite jet of control $u$ and let $x(\cdot)$ be the solution of (1) corresponding to $u$ and the initial condition $x(0)=x_{0}$. Then the delta derivative at $t=0$ of $\varphi(x(t), U(t))$ is equal to $\left(\delta_{\Sigma} \varphi\right)\left(x_{0}, U(0)\right)$.

The algebra $A(n, m)$ together with the operator $\delta_{\Sigma}$ is called the delta algebra of system $\Sigma$ and denoted by $A_{\Sigma}$. A homomorphism of delta algebras $A_{\Sigma}$ and $A_{\tilde{\Sigma}}$ is a homomorphism $\tau$ : $A(n, m) \rightarrow A(\tilde{n}, m)$ of algebras that satisfies the condition $\delta_{\tilde{\Sigma}} \circ \tau=\tau \circ \delta_{\Sigma}$. An isomorphism of the delta algebras $A_{\Sigma}$ and $A_{\tilde{\Sigma}}$ is a homomorphism that is a bijective map.

\section{THE MAIN RESULT}

The main result of this paper says the following

Theorem 4.1. Systems $\Sigma$ and $\tilde{\Sigma}$ are dynamically feedback equivalent iff their delta algebras $A_{\Sigma}$ and $A_{\tilde{\Sigma}}$ are isomorphic.

The proof of the above theorem rests on several propositions and lemmas stated below and to some extent follows the ideas of Jakubczyk (1992 and 1993) and Bartosiewicz et al. (1994).

Let $\eta=\left(\eta^{1}, \eta^{2}\right)$, where $\eta^{1}=\left(\eta_{1}, \ldots, \eta_{n}\right)$, $\eta^{2}=\left(\eta_{n+1}, \ldots, \eta_{n+m}\right)$ and $\eta_{s} \in A(\tilde{n}, m), s=$ $1,2, \ldots, n+m$.

Proposition 4. If two systems $\Sigma$ and $\tilde{\Sigma}$ are dynamically feedback equivalent via dynamic feedback transformations and $\eta^{1}$ and $\eta^{2}$ are defined by $\eta^{1}=\phi \circ \tilde{T}, \eta^{2}=\psi \circ\left(\tilde{T}, \tilde{P}_{J(m)}\right)$, then $\delta_{\tilde{\Sigma}} \eta^{1}=f \circ \eta$.

A similar fact holds for $\tilde{\eta}^{1}=\tilde{\phi} \circ T$ and $\tilde{\eta}^{2}=\tilde{\psi} \circ$ $(T, P)$, where $P=P_{J(m)}$. 
Let us define maps

$$
\begin{aligned}
& \hat{\eta}: \mathbb{R}^{\tilde{n}} \times J(m) \rightarrow \mathbb{R}^{n} \times J(m), \\
& \hat{\tilde{\eta}}: \mathbb{R}^{n} \times J(m) \rightarrow \mathbb{R}^{\tilde{n}} \times J(m)
\end{aligned}
$$

by $\hat{\eta}=\left(\eta^{1}, \eta^{2},\left(\delta_{\tilde{\Sigma}}^{j} \eta^{2}\right)_{j=1,2, \ldots}\right)$ and

$\hat{\tilde{\eta}}=\left(\tilde{\eta}^{1}, \tilde{\eta}^{2},\left(\delta_{\Sigma}^{j} \tilde{\eta}^{2}\right)_{j=1,2, \ldots}\right)$.

Consider the pullbacks

$\hat{\eta}^{*}(\varphi)=\varphi \circ \hat{\eta}, \hat{\eta}^{*}: A(n, m) \rightarrow A(\tilde{n}, m)$ and

$\hat{\tilde{\eta}}^{*}(\tilde{\varphi})=\tilde{\varphi} \circ \hat{\tilde{\eta}}, \hat{\tilde{\eta}}^{*}: A(\tilde{n}, m) \rightarrow A(n, m)$.

Proposition 5. If the assumptions of Proposition 4 are satisfied then the map $\hat{\eta}^{*}$ is an isomorphism of delta algebras $A_{\Sigma}$ and $A_{\tilde{\Sigma}}$ and $\left(\hat{\eta}^{*}\right)^{-1}=\hat{\tilde{\eta}}$.

Let $\tau: A_{\Sigma} \rightarrow A_{\tilde{\Sigma}}$ be a homomorphism of delta algebras. Let $x_{i}: \mathbb{R}^{n} \times J(m) \rightarrow \mathbb{R}$,

$u_{j}^{(k)}: \mathbb{R}^{n} \times J(m) \rightarrow \mathbb{R}$ be the coordinate functions

$$
\begin{gathered}
x_{i}(x, U)=x_{i}, i=1, \ldots, n, \\
u_{j}^{(k)}(x, U)=u_{j}^{(k)}, \\
j=1, \ldots, m, k=0,1, \ldots .
\end{gathered}
$$

Lemma 4.2. For any homomorphism

$$
\tau: A(n, m) \rightarrow A(\tilde{n}, m)
$$

of delta algebras there exists a unique map $\eta$ such that $\tau=\hat{\eta}^{*}$. Then $\tau\left(x_{i}\right)=\eta_{i}, i=1, \ldots, n$ and $\tau\left(u_{j}^{(0)}\right)=\eta_{n+j}, j=1, \ldots, m$.

In a similar way we can define the map

$\tilde{\eta}=\left(\tilde{\eta}_{1}, \ldots, \tilde{\eta}_{\tilde{n}+m}\right), \tilde{\eta}_{s} \in A(n, m), s=1, \ldots, \tilde{n}+m$ such that $\tilde{\eta}_{i}=\tilde{\tau}\left(x_{i}\right), \quad i=1, \ldots, \tilde{n}, \quad \tilde{\eta}_{\tilde{n}+j}=$ $\tilde{\tau}\left(u_{j}\right), j=1, \ldots, m$, where $\tilde{\tau}: A_{\tilde{\Sigma}} \rightarrow A_{\Sigma}$. This map also satisfies the last lemma.

\section{Sketch of proof of Theorem 4.1}

" $\Rightarrow$ " Assume that systems $\Sigma$ and $\tilde{\Sigma}$ are dynamically feedback equivalent. Then Proposition 5 gives the required isomorphism of difference algebras.

" $\Leftarrow$ " Assume that $\tau$ is an isomorphism between difference algebras $A_{\Sigma}$ and $A_{\tilde{\Sigma}}$. Let $\tau\left(x_{i}\right)=$ $\eta_{i}, i=1, \ldots, n, \tau\left(u_{j}^{(0)}\right)=\eta_{j+n}, j=1, \ldots, m$ and $\eta_{s} \in A(\tilde{n}, m) s=1, \ldots, n+m$. Take $\eta^{1}=\left(\eta_{1}, \ldots, \eta_{n}\right), \eta^{2}=\left(\eta_{n+1}, \ldots, \eta_{n+m}\right)$ and $\eta=$ $\left(\eta^{1}, \eta^{2}\right)$. From Lemma 4.2 it follows that $\tau=\hat{\eta}^{*}$. We are going to construct maps $\phi, \psi, \tilde{\phi}$ and $\tilde{\psi}$. Let $\phi=\left(\phi_{1}, \ldots, \phi_{n}\right)$ and $\psi=\left(\psi_{1}, \ldots, \psi_{m}\right)$.

First we assume that $(\tilde{X}, \tilde{U})$ is the jet of a trajectory of $\tilde{\Sigma}$ starting at 0 and we construct $\phi, \psi$ on jets of trajectories, only. Define

$$
\phi_{i}(\tilde{X}):=\left(\tau x_{i}\right)\left(\tilde{T}^{-1}(\tilde{X})\right), \quad i=1, \ldots, n,
$$

and

$$
\psi_{j}(\tilde{X}, \tilde{U}):=\left(\tau u_{j}^{(0)}\right)\left(\tilde{T}^{-1}(\tilde{X})\right), \quad j=1, \ldots, m .
$$

One can show that $(X, U)$ defined by $X=\Phi(\tilde{X})$ and $U=\Psi(\tilde{X}, \tilde{U})$ is the jet of a trajectory of $\Sigma$ starting at 0 .

To finish the proof it is enough to extend the maps $\phi, \psi, \tilde{\phi}$ and $\tilde{\psi}$ to arbitrary $(\tilde{X}, \tilde{U}) \in J(\tilde{n}) \times J(m)$ and $(X, U) \in J(n) \times J(m)$. This can be done similarly as in (Jakubczyk, 1993).

\section{CONCLUSION}

The paper contains a characterization of dynamic feedback equivalence of nonlinear control systems defined on homogeneous time scales. Namely, two systems are dynamically feedback equivalent if and only if their delta algebras are isomorphic. This theorem is a generalization of earlier results stated for continuous-time systems and discretetime systems. It contains these results as particular cases. If the time scale is the real line, then the delta algebra of the system becomes the differential algebra of the continuous-time system. On the other hand, if the time scale is the set of integer numbers, then the delta algebra of the system is related to the difference algebra of the discrete-time system.

Dynamic feedback equivalence is close to the idea of dynamic feedback linearization. A nonlinear control system is dynamically feedback linearizable if it is dynamically feedback equivalent to a linear controllable system. The methods used for studying dynamic feedback linearization for continuoustime systems and for discrete-time systems may now be generalized to systems defined on homogeneous time scales.

\section{REFERENCES}

Bartosiewicz, Z., Jakubczyk, B. and Pawłuszewicz, E. (1994). Dynamic feedback equivalence of nonlinear discrete-time systems. In: Proceedings of First International Symposium on Mathematical Models in Automation and Robotics, Sept. 1-3, 1994, Miȩdzyzdroje, Poland, Tech. Univ. of Szczecin Press, Szczecin.

Bartosiewicz, Z. and Pawłuszewicz, E. (1998). External equivalence of unobservable discretetime systems. In: Proceedings of NOLCOS'98, Enschede, Netherlands, July 1998.

Bartosiewicz, Z. and Pawłuszewicz, E. (2004a). Unification of continuous-time and discretetime systems: the linear case. In Proceedings of Sixteenth International Symposium on Mathematical Theory of Networks and Systems (MTNS2004) Katholieke Universiteit Leuven, Belgium July 5-9, 2004, Leuven. 
Bartosiewicz, Z. and Pawłuszewicz, E. (2004b). Linear control systems on time scales: unification of continuous and discrete. In: Proceedings of 10th IEEE International Conference on Methods and Models in Automation and Robotics (MMAR2004), 30 August - 2 September 2004, Miȩdzyzdroje, Poland.

Bohner, M. and Peterson, A. (2001). Dynamic Equations on Time Scales, Birkhauser, Boston.

Fliess, M., Lévine, J., Martin, Ph. and Rouchon, P. (1992) Sur les systemes non linaires diffrentiellement plats. C. R. Acad. Sci. Paris Sr. I Math. 315, no. 5, 619-624.

Fliess, M., Lévine, J., Martin, Ph. and Rouchon, P. (1993) Linarisation par bouclage dynamique et transformations de Lie-Bcklund. C. R. Acad. Sci. Paris Sr. I Math. 317, no. 10, 981-986.

Goodwin, G.C., Graebe, S.F. and Salgado, M.E. (2001). Control System Design, Prentice Hall International.

Hilger, S. (1988). Ein Maßkettenkalkül mit Anwendung auf Zentrumsmannigfaltigkeiten, Ph.D. thesis, Universität Würzburg.

Jakubczyk, B. (1992). Remarks on equivalence and linearization of nonlinear systems. In: Proceedings of the 2nd IFAC NOLCOS Symposium, 1992, Bordeaux, France, 393-397.

Jakubczyk, B. (1993) Dynamic feedback equivalence of nonlinear control systems, unpublished manuscript.

Marino, R. (1990)Static and dynamic feedback linearization of nonlinear systems. In: Perspectives in control theory (Sielpia, 1988), Progr. Systems Control Theory, 2, Birkhuser Boston, Boston, MA, 249-260.

Monaco, S. and Normand-Cyrot, D. (1995). A unified representation for nonlinear discrete-time and sampled dynamics, Journal of Mathematical Systems, Estimation, and Control, vol. 5, 1-27.

Pawłuszewicz, E. and Bartosiewicz, Z. (1999). External dynamic feedback equivalence of observable discrete-time control systems. In: Differential Geometry and Control, Proceedings of Symposia in Pure Mathematics, vol. 64, (Eds. G. Ferreyra, R. Gardner, H. Hermes and H. Sussmann), Americam Mathematical Society, Providence.

Pomet, J.-B. (1995) A differential geometric setting for dynamic equivalence and dynamic linearization. In: Geometry in Nonlinear Control and Differential Inclusions, Banach Center Publication, vol.32, Warsaw, 319-339. 